\title{
Bilayer Electrospun Nanofibre Structures to Improve Quality Factor in Air Filtration
}

\author{
Vinod Kadam ${ }^{1,2}$, Yen Bach Truong ${ }^{2}$, Ilias Louis Kyratzis ${ }^{2}$, Lijing Wang ${ }^{1}$, Rajiv Padhye ${ }^{1}$ \\ ${ }^{1}$ School of Fashion \& Textiles, RMIT University \\ Brunswick, VIC, 3056, Australia \\ ${ }^{2}$ CSIRO-Manufacturing \\ Clayton, VIC, 3168, Australia
}

\begin{abstract}
Electrospun nanofibres have been used in air filtration applications ranging from indoor filters to personal protective respirators. Nanofibres based filter exhibit excellent filtration efficiency but high pressure drop. The function of filtration efficiency and pressure drop is expressed by a term called quality factor. This study identified a method to improve the quality factor of layered nanofibre structures by reducing the pressure drop. Single and bilayer structures from polyacrylonitrile nanofibres of equal mass coverage were produced by optimising electrospinning time. Further, the stacking order of nanofibres in the bilayer structure was altered and compared. This alteration changed the surface morphology of the nanofibre structures influencing fibre diameter, pore size, and pore size distribution. The fibre diameter and pore size were determined using field emission scanning electron microscope (FESEM) and capillary flow porometer, respectively. The thickness of the nanofibre structure was measured using laser microscopy. The thickness and packing density of single layer and bilayer nanofibre structures differed despite equal mass coverage. Furthermore, change in the layering order of bilayer nanofibre structures also had an effect on packing density which consequently affected the filtration efficiency and pressure drop. A bilayer structure with $400 \mathrm{~nm}$ average fibre diameter at the top covering $200 \mathrm{~nm}$ beneath considerably reduced the pressure drop as compared to its single structure counterparts. This bilayer nanofibre stacking arrangement was also improved quality factor significantly as compared to that of single layer structures.
\end{abstract}

Keywords: Electrospinning; Air filtration; Pressure drop; Quality factor; Bilayer structure

\section{Introduction}

Electrospun nanofibres produce excellent air filter media owing to their nanosize fibre diameters, small pore size, interconnected pore structure, high specific surface area and low mass coverage (basis weight) [1-4]. They have been commercially successful in a range of filtration applications such as particulate filter and high-efficiency air filter [5]. Electrospun nanofibres capture particulate matter (tiny aerosols of size 50-5000 nm) in the air due to effective diffusion and interception mechanism [6,7]. Diffusion is a mass transfer of fine aerosols $(<500 \mathrm{~nm})$ due to random movement even at low air flow whereas interception is capturing the aerosol particles at the nanofibre surface by reducing the distance between fibre surface and aerosol centre.

Air filter performance is mostly measured in terms of filtration efficiency, pressure drop and quality factor [8]. The filtration efficiency is the ratio of particle concentration upstream (before) and downstream (after) the filtration. Pressure drop is the air pressure difference upstream to downstream. A combination of high filtration efficiency and low pressure drop produce the best filter, especially for a respiratory air filter; however, filtration efficiency and pressure drop are inversely related to each other. The quality factor of a filter which is a combination of filtration efficiency and pressure drop makes a balance between the two. Different multilayer structures $[9,10]$ with variable mass coverage and packing density have been fabricated to improve air filtration performance, credit to versatility and tunable morphologies of electrospun nanofibres. The multilayer structures proved beneficial over single layer structures in reducing the pressure drop and producing high quality factors. Each layer was stacked on an individual nonwoven substrate [9] which elevates the total weight of the filter media. Instead, bilayer structures on a single nonwoven substrate can also serve the same purpose. Besides this, nanofibre diameter and its stacking arrangement in bilayer structure can also be tuned to compare the effect of the pressure drop on to the quality factor. 
This paper reports on the filtration performance of single layer and bilayer nanofibre structures. The effect of nanofibre layers and its alternate stacking order on surface morphology, thickness, and packing density is examined. The improvement in the quality factor due to the specific stacking of the nanofibres in bilayer structure is reported.

\section{Material and Methods}

Polyacrylonitrile (PAN) (MW 150, $000 \mathrm{~g} / \mathrm{mol}$ ) polymer was purchased from Sigma-Aldrich, USA, and N, Ndimethylformamide (DMF) (99.7\%) solvent from Merck. The polymer and solvent were used as received without any further purification. PAN (8 wt $\%$ and $10 \mathrm{wt} \%$ ) was dissolved in DMF by stirring for twelve hours at room temperature. The polyester-viscose nonwoven substrate was used to support PAN nanofibres prepared by electrospinning.

\subsection{Electrospinning}

Figure 1 shows the electrospinning setup used in this study. A $5 \mathrm{ml}$ syringe (Terumo) containing PAN solution was placed on the syringe pump (NE1000, New Era Pump Systems, Inc. USA). The flow rate of the solution was 0.5 $\mathrm{ml} / \mathrm{hr}$. A metallic needle (18 gauge) was attached to the syringe. A DC voltage power supply (Spellman SL150, USA) of $18 \mathrm{kV}$ was applied to the tip of the needle. The distance between needle tip and the collector drum was $20 \mathrm{~cm}$. The nanofibres were collected on the collector drum (a metal surface roller of $13 \mathrm{~cm}$ diameter) rotating at $180 \mathrm{rpm}$. The drum was connected to a ground electrode.

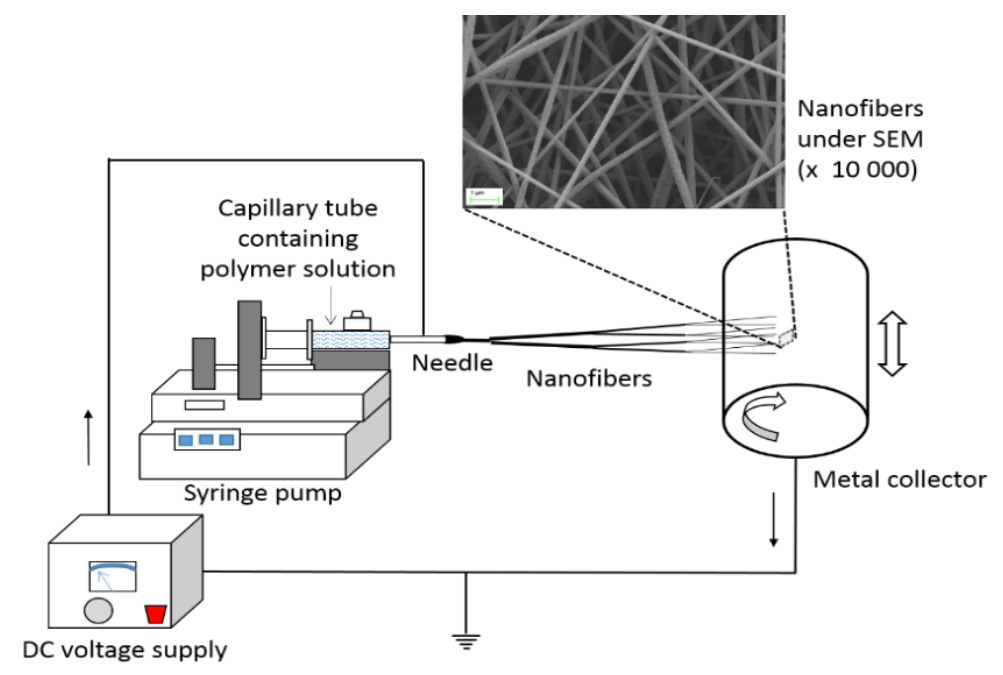

Fig. 1: Electrospinning set up comprised of the high voltage supply, syringe pump and grounded collector drum.

\subsection{Single and Bilayer Nanofibre Structures}

The single layer and bilayer nanofibre structures with equivalent mass coverage were prepared from electrospun nanofibres. The electrospinning time (nanofibre deposition time) varied from 96-120 minutes to achieve uniform mass coverage of the single layer and bilayer structures. The mass coverage was estimated by multiplying the polymer concentration with the feed rate and electrospinning time over the collector area of $19 \times 34 \mathrm{~cm}$. The mass coverage of $1.2 \mathrm{~g} / \mathrm{m}^{2}$ was kept constant for all the structures. Table 1 presents electrospinning parameters, nanofibre diameters and layering order for single and bilayer nanofibre structures. 
Table 1: Details of the electrospun single and bilayer nanofibre structure formation with identical mass coverage of $1.2 \mathrm{~g} / \mathrm{m}^{2}$.

\begin{tabular}{|c|c|c|c|c|}
\hline Layers & Single layer & Single layer & Bilayer & Bilayer \\
\hline Sample code & S8 & S10 & B8 & B10 \\
\hline PAN concentration $(\%)(\mathrm{w} / \mathrm{w})$ & 8 & 10 & 8 and 10 & 8 and 10 \\
\hline Electrospinning time (minutes) & 120 & 96 & 108 & 108 \\
\hline Nanofibre diameter $(\mathrm{nm})$ & $\approx 200$ & $\approx 400$ & $\approx 200$ on top & $\approx 400$ on top \\
\hline \multirow[t]{2}{*}{ Layering order } & \multirow[t]{2}{*}{$200 \mathrm{~nm}$} & \multirow[t]{2}{*}{$400 \mathrm{~nm}$} & $200 \mathrm{~nm}$ & $400 \mathrm{~nm}$ \\
\hline & & & $400 \mathrm{~nm}$ & $200 \mathrm{~nm}$ \\
\hline
\end{tabular}

\subsection{Nanofibre Morphology}

The nanofibre samples were iridium coated using a Cressington 208 HRD iridium sputter coater and imaged at $3 \mathrm{kV}$ by Field Emission Scanning Electron Microscope (FE-SEM) (Zeiss Gemini 2 Merlin). The diameter was measured using the Image-J software. The thickness of all the structures was measured using a non-contact 3D laser scanning digital microscope (Olympus LEXT OLS 4100) by determining the height difference between nanofibre structure and the nonwoven substrate over an average area of $0.5 \mathrm{~mm} 2$ at $\mathrm{X} 5$ magnification. The packing density was estimated by following Eq. (1):

$$
\alpha=\frac{m}{z \times a \times \rho}
$$

Where, $\alpha$ is the packing density, $\mathrm{m}, \mathrm{z}$ and a are the estimated mass coverage, thickness and area respectively, and $\rho$ is the density of PAN (1.184 gm/cm3). The pore diameter and the pore size distribution of each membrane (13 mm diameter) were determined using a capillary flow porometer (Porous Materials Inc, USA).

\subsection{Air Filtration Performance}

Figure 2 shows the experimental set up to measure air filtration performance of nanofibre structures. HEPA filters at both ends of the duct ensured that the ducts were clean all the time. Potassium chloride $(\mathrm{KCl})$ aerosol particles $(0.3-5 \mu \mathrm{m}$ diameter) were generated by an atomizer and directed towards the sample. The effective test area of $93.26 \mathrm{~cm} 2 \mathrm{was}$ tested against the $\mathrm{KCl}$ aerosols at the face velocity of $0.06 \mathrm{~m} / \mathrm{s}$ (flow rate of $20 \mathrm{~L} / \mathrm{min}$ ) and $0.08 \mathrm{~m} / \mathrm{s}$ (flow rate of $32 \mathrm{~L} / \mathrm{min}$ ). The upstream and downstream aerosol concentration was measured by optical particle counters (PMS HandiLaz). Pressure drop was measured by a differential pressure sensor upstream and downstream from the sample. Filtration test properties were reported as the average of five replicate samples. Air filtration performance was determined in terms of filtration efficiency, pressure drop and quality factor, as shown in Eqs. (2) - (4) below: 


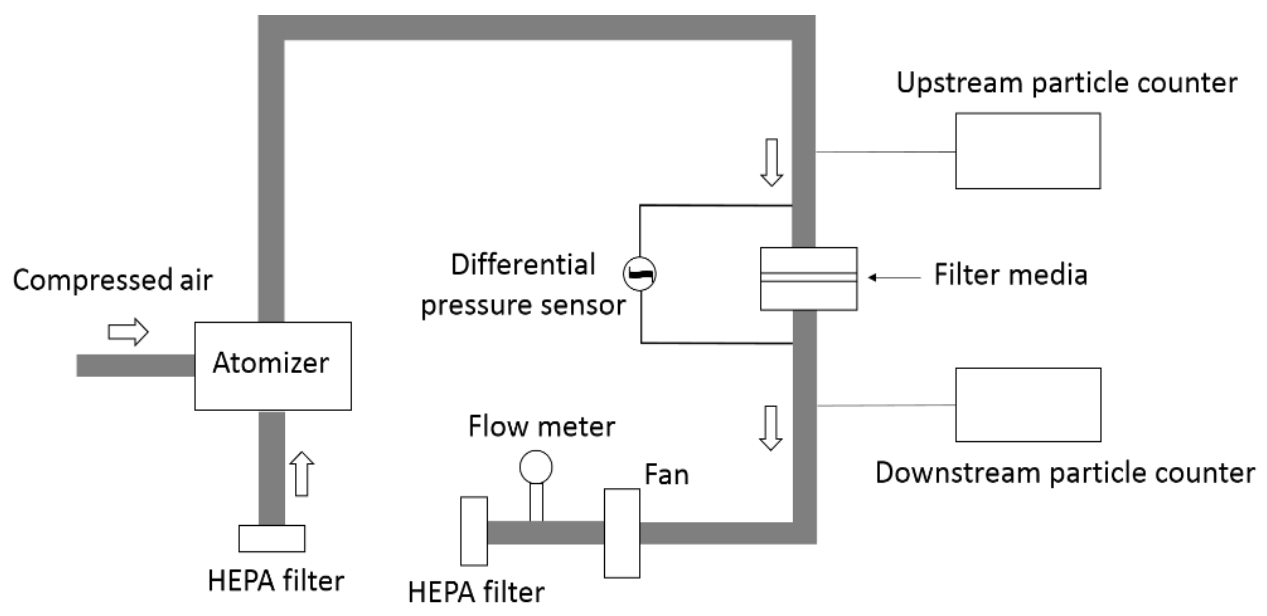

Fig. 2: Air filtration measurement set up for aerosols of size $0.3-5 \mu \mathrm{m}$.

$$
\begin{gathered}
\eta=\frac{\mathrm{n}_{1}-\mathrm{n}_{2}}{\mathrm{n}_{1}} \\
\Delta \mathrm{P}=P_{1}-P_{2} \\
\mathrm{Q}=\frac{-\operatorname{In}(1-\eta)}{\Delta \mathrm{p}}
\end{gathered}
$$

Where: $\eta$ represents the filtration efficiency; $\mathrm{n}_{1}$ represents the upstream aerosol particle concentration; $\mathrm{n}_{2}$ represents the downstream aerosol particle concentration; $\Delta \mathrm{P}$ represents the pressure drop; $\mathrm{P}_{1}$ represents the upstream air absolute pressure; $\mathrm{P}_{2}$ represents the downstream air absolute pressure and; $\mathrm{Q}$ represents the quality factor.

\section{Results and discussion}

\subsection{Nanofibre diameter}

Figure $3 \mathrm{a}$ and $3 \mathrm{~b}$ shows SEM images of the single layer electrospun nanofibres produced from $8 \mathrm{wt} \%$ and $10 \mathrm{wt} \%$ PAN concentration with an average diameters of $197 \pm 26 \mathrm{~nm}$ and $397 \pm 38 \mathrm{~nm}$ respectively. As can be seen, nanofibre diameter is influenced by the polymer concentration. Fibres produced under identical electrospinning conditions, the higher polymer concentration resulted into larger nanofibre diameters. Figure $3 \mathrm{c}$ and $3 \mathrm{~d}$ presents the top surface of bilayer nanofibre structures. As expected, the B8 structure resembles the S8 structure while B10 resembles S10. This resemblance is logical since the nanofibre layers were prepared from S8 and S10 structures using similar electrospinning parameters.

In all the SEM images, micro-scale roughness is prominent at the surface of PAN nanofibres unlike the rod-like smooth nanofibres reported in other studies $[11,12]$. This roughness may be due to the different accelerating voltages used to view the samples under SEM. A low accelerating voltage $(3 \mathrm{kV})$ utilized in this study whereas other reports used higher accelerating voltages from $5-15 \mathrm{kV}$. 

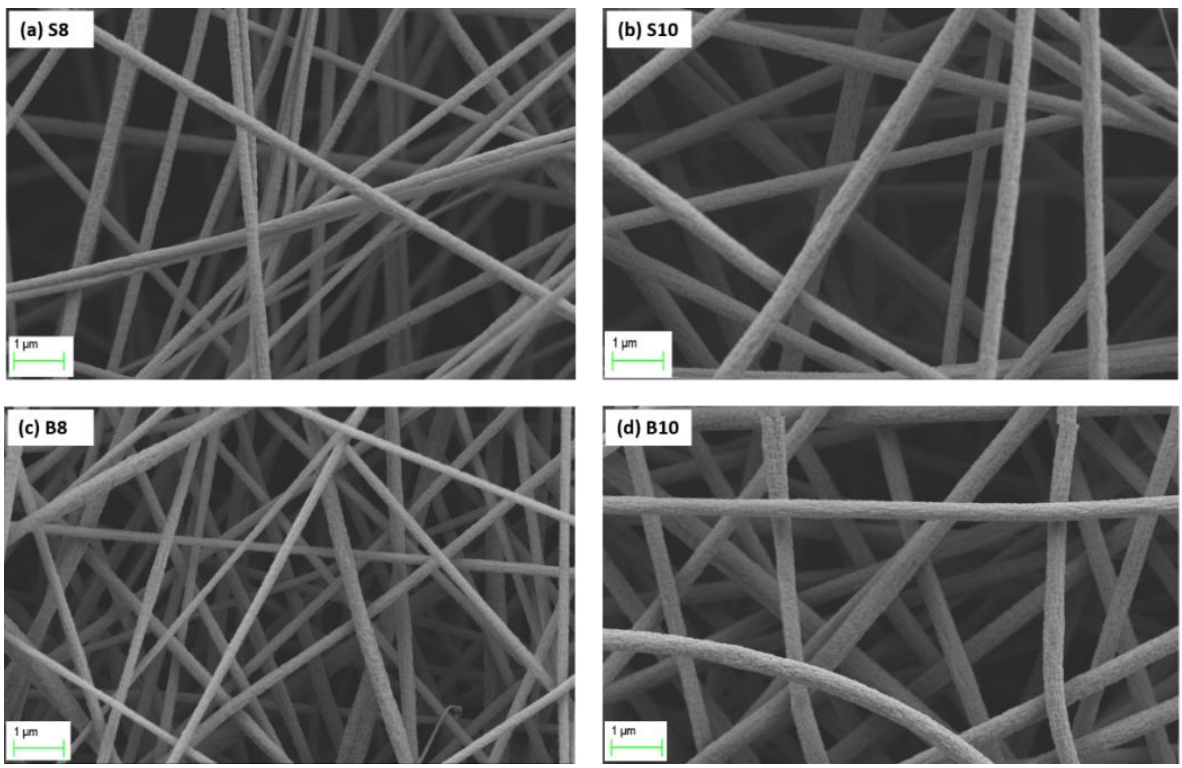

Fig. 3: SEM images of (a) S8; (b) S10; (c) B8; and (d) B10 nanofibre structures at X 10,000.

\subsection{Pore Size}

Smaller fibre diameter in a filter reduces its pore size and porosity [13]. In this context, the pore size represents vacant space between the fibres resulting in capillaries throughout the fibre structure. The smaller pores lead to increased filtration efficiency but also elevated pressure drop [3]. The pore size of the single and bilayer nanofibre structures at $1.2 \mathrm{~g} / \mathrm{m} 2 \mathrm{mass}$ coverage laid on the nonwoven substrate is presented in Table 2 . The average pore size of S8 is the smallest $(1.6 \mu \mathrm{m})$ due to the smaller fibre diameter $(\approx 200 \mathrm{~nm})$ than $\mathrm{S} 10(\approx 400 \mathrm{~nm})$ structure. The pore size results of S8 and B8 are comparable. Similarly, S10 and B10 showed pore sizes likewise. It is the nanofibres at the top surface that largely influences the average pore size. The minimum, maximum and average pore size of B10 were slightly smaller as compared to S10. This reduction is because of the presence of $200 \mathrm{~nm}$ fibre layer under the $400 \mathrm{~nm}$ fibres which does change the pore size distribution in single and bilayer nanofibre structures.

Table 2: Pore size of single and bilayer nanofibre structures at $1.2 \mathrm{~g} / \mathrm{m}^{2}$ mass coverage.

\begin{tabular}{|c|c|c|c|}
\hline Sample & \multicolumn{3}{|c|}{ Pore size $(\boldsymbol{\mu m})$} \\
\hline & Minimum & Maximum & Average \\
\hline S8 & 1.5 & 2.6 & 1.6 \\
\hline S10 & 2.4 & 3.7 & 2.5 \\
\hline B8 & 1.6 & 2.9 & 1.7 \\
\hline B10 & 2 & 3.5 & 2.1 \\
\hline
\end{tabular}

The pore size distribution also has an effect on filtration performance [14]. Figure 4 displays pore size distribution of the single and bilayer nanofibre structures at $1.2 \mathrm{~g} / \mathrm{m} 2$ mass coverage. It indicates that the pore size distribution of the bilayer structures is somewhere in between the S8 and S10 single layer structures. Single layer structures exhibited relatively narrow pore size distribution. This difference in pore size distribution is logical since the bilayer structures are fabricated from two different sizes of nanofibre diameter. 


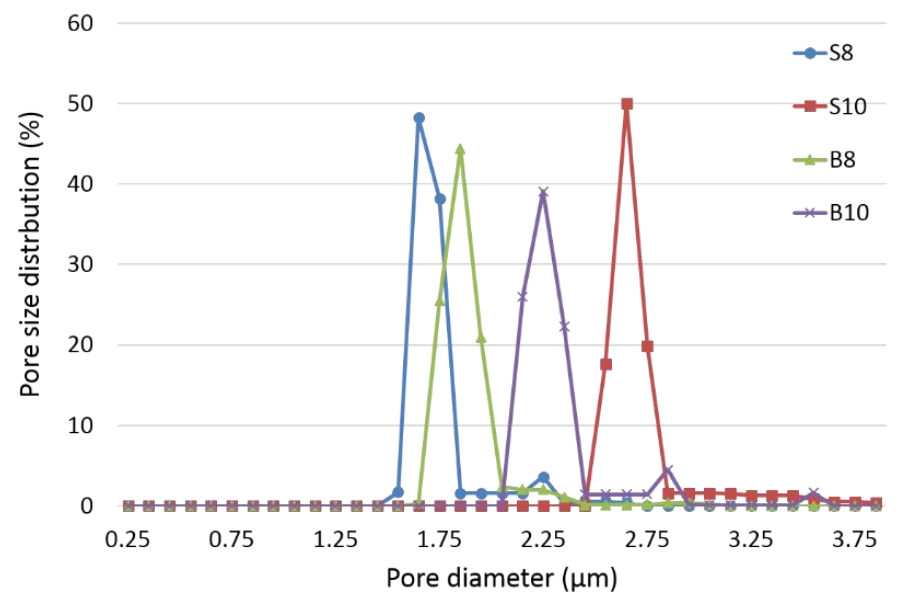

Fig. 4: Pore size distribution of single and bilayer nanofibre structures at $1.2 \mathrm{~g} / \mathrm{m}^{2}$ mass coverage.

\subsection{Thickness and Packing Density}

All nanofibre structures recorded different thickness values despite equal mass per unit area (Table 3). The single layer S8 showed the smallest thickness $(118 \mu \mathrm{m})$ whereas S10 exhibited the highest thickness $(218 \mu \mathrm{m})$. This difference in the thicknesses is attributed to the modification in fibre diameter of S8 and S10 structures. $400 \mathrm{~nm}$ fibre in the S10 can be thicker than $200 \mathrm{~nm}$ fibres in the S8 structure. Unexpectedly, the bilayer B8 and B10 structures did not show equivalent thickness. B10 is thinner than B8 by around $31 \mu \mathrm{m}$. This reduction in thickness is due to the compressing action of thick nanofibres over relatively thin nanofibres. In the case of B10, the thicker $400 \mathrm{~nm}$ fibres are at the top of the structure and can easily compress the thinner $200 \mathrm{~nm}$ fibres underneath. Whereas, it is a lot harder for the thinner fibres to compress or squeeze the thicker $400 \mathrm{~nm}$ fibres in the B8 structure.

The packing density of a nanofibre structure also has more prominent on air filtration than the thickness [9]. The packing density of single layer structures decreased from $8.59 \times 10^{-3}$ to $4.65 \times 10^{-3}$ when the thickness increased from 118 to $218 \mu \mathrm{m}$ (Table 3 ). The bilayer B8 structure shows relatively less packing density $\left(5.20 \times 10^{-3}\right)$ than B10 structure $\left(6.18 \times 10^{-3}\right)$ due to the larger overall thickness of the B8 structure at an equal mass coverage of $1.2 \mathrm{~g} / \mathrm{m}^{2}$.

Table 3: Thickness and packing density of nanofibre structures at $1.2 \mathrm{~g} / \mathrm{m}^{2}$ mass coverage.

\begin{tabular}{|l|c|c|}
\hline Sample & Thickness $(\boldsymbol{\mu m})$ & Packing density $\left.(\mathbf{x ~ 1 0})^{-3}\right)$ \\
\hline S8 & $118 \pm 6$ & 8.59 \\
\hline S10 & $218 \pm 25$ & 4.65 \\
\hline B8 & $195 \pm 13$ & 5.20 \\
\hline B10 & $164 \pm 20$ & 6.18 \\
\hline
\end{tabular}

\subsection{Filtration Efficiency and Pressure Drop}

Figure 5 presents the filtration performance of the nanofibre structures at face velocities of $0.06 \mathrm{~m} / \mathrm{s}$ and $0.08 \mathrm{~m} / \mathrm{s}$. It is worth to mention that the nanofibre layered structures faced the air flow during the filtration and not the nonwoven substrate. The single layer S8 showed the highest pressure drop whereas the bilayer B10 structure significantly reduced the pressure drop as compared to all other structures. The pressure drop of B10 in relation to S8 reduced from $145 \mathrm{~Pa}$ to $64 \mathrm{~Pa}$ at $0.06 \mathrm{~m} / \mathrm{s}$ velocity and from $208 \mathrm{~Pa}$ to $87 \mathrm{~Pa}$ at $0.08 \mathrm{~m} / \mathrm{s}$ face velocity, respectively. This reduction in pressure drop is attributed to two things. First, the packing density of B10 $\left(6.18 \times 10^{-3}\right)$ is lower than in $\mathrm{S} 8\left(8.59 \times 10^{-3}\right)$ and second, the pore size of B10 $(2.1 \mu \mathrm{m})$ is higher than $\mathrm{S} 8(1.6 \mu \mathrm{m})$. However, the structure B8 showed higher pressure drop (149 Pa at $0.08 \mathrm{~m} / \mathrm{s}$ face velocity) compared to B10 (87 Pa) despite having lower packing density. This increase in pressure drop may be because B8 had a small pore size $(1.7 \mu \mathrm{m})$ and uniform pore size distribution than B10 $(2.1 \mu \mathrm{m})$. Hence, it is found that in bilayer nanofibre structures, the relationship between pore size and packing density is not straightforward, unlike in single layer structures. Another reason for the decreased 
pressure drop in the bilayer B10 structure could be due to the decreased tortuosity through the sample in relation to the B8 structure. Air can easily flow through the coarse $400 \mathrm{~nm}$ fibres compared to the finer $200 \mathrm{~nm}$ fibres at the surface.

The filtration efficiency was found highest (98.5\%) for the single layer S8 structure which is attributed to $200 \mathrm{~nm}$ fibre diameter and small pore size. The filtration efficiency of the bilayer B8 (92.4\%) and B10 (93.5\%) structure was comparable at $0.08 \mathrm{~m} / \mathrm{s}$ face velocity; however, at $0.06 \mathrm{~m} / \mathrm{s}$ face velocity, it was found lower for B10 (90.6\%) compared to B8 (96.8\%). There was no specific trend observed in the results for filtration efficiency, unlike in the case of pressure drop for the different nanofibre structures.

(a)

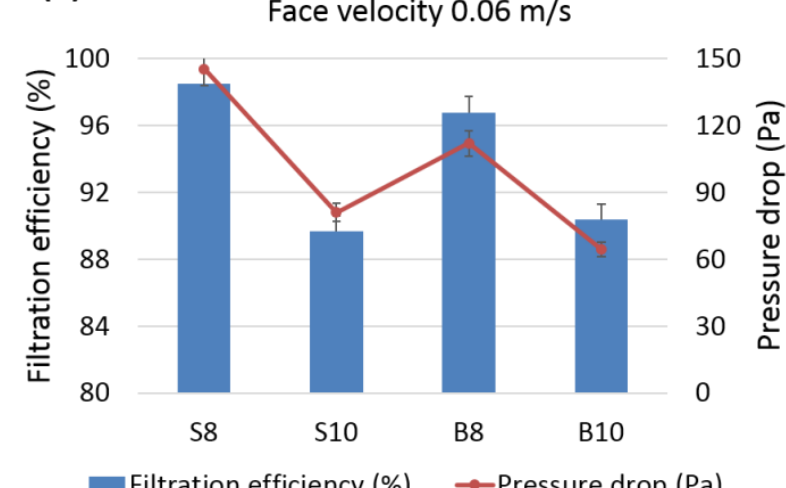

(b)

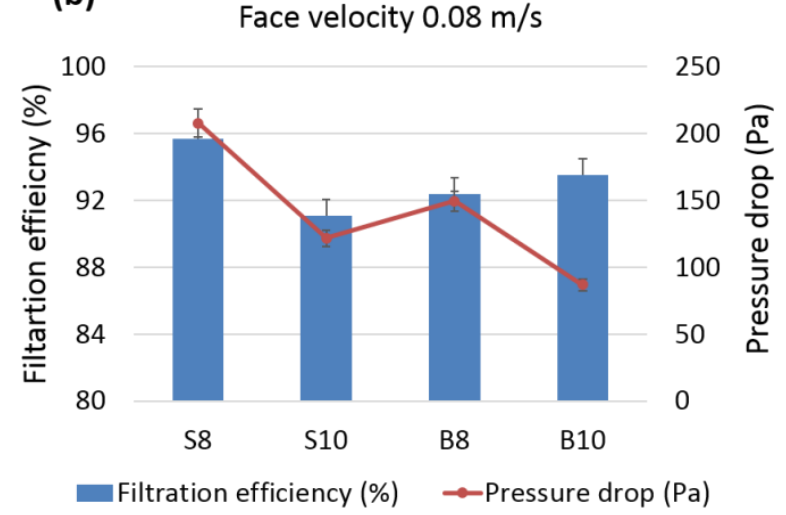

Fig. 5: Filtration efficiency and pressure drop comparison of nanofibre layered structures.

\subsection{Quality Factor}

The quality factor is a better indicator of filtration performance as it is a function of both filtration efficiency and pressure drop. Figure 6 shows quality factor values for S8, S10, B8 and B10 at the face velocities of $0.06 \mathrm{~m} / \mathrm{s} \mathrm{and} 0.08 \mathrm{~m} / \mathrm{s}$. The quality factor of all the structures was found to be better at the lower face velocity. Hence, low face velocity along with low mass coverage are recommended for better filtration performance regarding the quality factor [9]. At $0.06 \mathrm{~m} / \mathrm{s}$ face velocity, the quality factor values of S8 $(29.1 / \mathrm{kPa}), \mathrm{S} 10(28.1 / \mathrm{kPa})$ and B8 $(30.9 / \mathrm{kPa})$ were comparable. The bilayer B10 structure outperformed all other structures and showed highest quality factor $36.3 / \mathrm{kPa}$ and $31.4 / \mathrm{kPa}$ for face velocities of $0.06 \mathrm{~m} / \mathrm{s}$ and $0.08 \mathrm{~m} / \mathrm{s}$, respectively. This finding implies that the stacking order of nanofibres in a bilayer structure has a profound effect on the quality factor. Furthermore, the quality factor of bilayer B10 structure $(36.9 / \mathrm{kPa})$ was found better than the PAN transparent filter recently reported in the literature $(24 / \mathrm{kPa})[15]$.

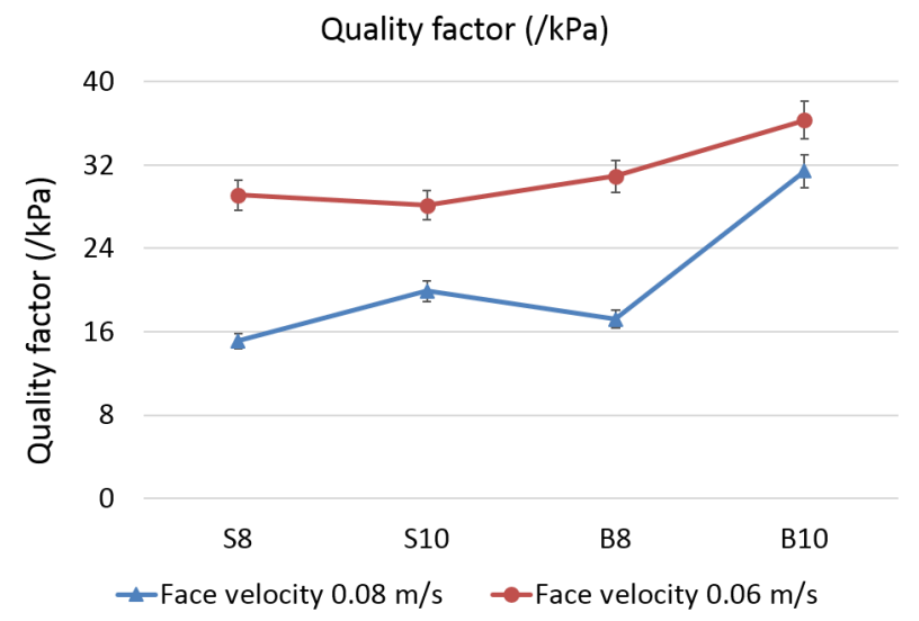

Fig. 6: The quality factor of nanofibre layered structures at face velocities of $0.08 \mathrm{~m} / \mathrm{s}$ and $0.06 \mathrm{~m} / \mathrm{s}$ with $1.2 \mathrm{~g} / \mathrm{m}^{2}$ mass coverage. 


\section{Conclusion}

Air filtration performance of single layer and bilayer nanofibre structures is presented in this research paper. The bilayer nanofibre structure fabricated from two single layers of nanofibres composed of $200 \mathrm{~nm}$ and $400 \mathrm{~nm}$ diameter fibres. It is found that the morphology of bilayer structure is influenced by the order of the single layer structures. The fibre diameter and pore size characteristics of bilayer structure is dominated by its top surface layer. The bilayer nanofibre structure shows different thickness and packing density even at equivalent mass coverage as compared to single layers. The stacking order in the bilayer structure also influenced the filtration performance. The bilayer structure with $400 \mathrm{~nm}$ fibre diameter at the top and $200 \mathrm{~nm}$ fibre diameter at the bottom showed a significant reduction in pressure drop (from $208 \mathrm{~Pa}$ to $87 \mathrm{~Pa}$ ) but comparable filtration efficiency. Furthermore, the quality factor is also improved due to this specific stacking order in the bilayer structure. This bilayer structure formation is easy to fabricate and scalable. Such an approach can be practically used in respiratory air filtration where less pressure drop is desirable.

\section{Acknowledgements}

Vinod Kadam acknowledges RMIT University, Australia for International Scholarship towards PhD study, CSIRO, Australia for providing facilities and materials to carry out the research and Indian Council of Agricultural Research, India for granting study leave. Thanks to Jurg Schutz for assisting in air filtration measurements.

\section{References}

[1] F. K. Ko and Y. Wan, Introduction to nanofiber material. Cambridge University Press, 2014.

[2] S. Sundarrajan, K. L. Tan, S. H. Lim, and S. Ramakrishna, "Electrospun nanofibers for air filtration applications," Procedia Engineering, vol. 75, pp. 159-163, 2014.

[3] V. Kadam, L. Wang, and R. Padhye, "Electrospun nanofibre materials to filter air pollutants - A review," Journal of Industrial Textiles, 2016.

[4] X. Li, N. Wang, G. Fan, J. Yu, J. Gao, G. Sun, et al., "Electreted polyetherimide-silica fibrous membranes for enhanced filtration of fine particles," Journal of Colloid and Interface Science, vol. 439, pp. 12-20, 2015.

[5] R. S. Barhate and S. Ramakrishna, "Nanofibrous filtering media: Filtration problems and solutions from tiny materials," Journal of Membrane Science, vol. 296, pp. 1-8, 2007.

[6] B. Maze, T. H. Vahedi, Q. Wang, and B. Pourdeyhimi, "A simulation of unsteady-state filtration via nanofiber media at reduced operating pressures," Journal of Aerosol Science, vol. 38, pp. 550-571, 2007.

[7] H. Wang, G. Zheng, X. Wang, and D. Sun, "Study on the air filtration performance of nanofibrous membranes compared with conventional fibrous filters," in Nano/Micro Engineered and Molecular Systems (NEMS), 2010 5th IEEE International Conference London, UK, pp. 387-390, 2010.

[8] J. R. Sanchez, J. M. Rodriguez, A. Alvaro, and A. M. Estevez, "Comparative study of different fabrics in the filtration of an aerosol using more complete filtration indexes," Filtration and Separation, vol. 34, pp. 593-598, 1997.

[9] W. W. F. Leung, C. H. Hung, and P. T. Yuen, "Effect of face velocity, nanofiber packing density and thickness on filtration performance of filters with nanofibers coated on a substrate," Separation and Purification Technology, vol. 71, pp. 30-37, 2010.

[10] C. H. Hung and W. W. F. Leung, "Filtration of nano-aerosol using nanofiber filter under low Peclet number and transitional flow regime," Separation and Purification Technology, vol. 79, pp. 34-42, 2011.

[11] J. Matulevicius, L. Kliucininkas, T. Prasauskas, D. Buivydiene, and D. Martuzevicius, "The comparative study of aerosol filtration by electrospun polyamide, polyvinyl acetate, polyacrylonitrile and cellulose acetate nanofiber media," Journal of Aerosol Science, vol. 92, pp. 27-37, 2016.

[12] Y. Liu, M. Park, B. Ding, J. Kim, M. El-Newehy, S. S. Al-Deyab, et al., "Facile electrospun polyacrylonitrile/poly(acrylic acid) nanofibrous membranes for high efficiency particulate air filtration," Fibers and Polymers, vol. 16, pp. 629-633, 2015.

[13] S. Borhani, S. A. Hosseini, S. G. Etemad, and J. Militký, "Structural characteristics and selected properties of polyacrylonitrile nanofiber mats," Journal of Applied Polymer Science, vol. 108, pp. 2994-3000, 2008. 
[14] N. Wang, Z. Zhu, J. Sheng, S. S. Al-Deyab, J. Yu, and B. Ding, "Superamphiphobic nanofibrous membranes for effective filtration of fine particles," Journal of Colloid and Interface Science, vol. 428, pp. 41-48, 2014.

[15] C. Liu, P. C. Hsu, H. W. Lee, M. Ye, G. Zheng, N. Liu, et al., "Transparent air filter for high-efficiency PM2. 5 capture," Nature communications, vol. 6, p. 6205, 2015. 